\title{
Suspicion of pulmonary embolism during treatment of pemphigoid gestationis
}

\author{
Magdalena Podolec-Rubiś, Marta Wołek, Paweł Brzewski, Anna Wojas-Pelc
}

Department of Dermatology, Jagiellonian University Medical College, Krakow, Poland

Head: Prof. Anna Wojas-Pelc MD, PhD

Postep Derm Alergol 2013; XXX, 1: 59-61

DOI: 10.5114/pdia.2013.33381

\begin{abstract}
Pemphigoid gestationis (PG), first described in 1872 by Laws Milton, is a rare autoimmune bullous disease of pregnancy and puerperium. Pemphigoid gestationis has been estimated to occur in 1:50 000 pregnancies and usually presents in the second or third trimester with exacerbation after partum. This disease is caused by circulating IgG1 immunoglobulin against hemidesmosomal protein bullous pemphigoid BP180 (BPAG2) type XVII collagen and less frequently BP230. We present a case of pemphigoid gestationis in primigravida with complications during treatment due to the suspicion of pulmonary embolism.
\end{abstract}

Key words: pemphigoid gestationis, herpes gestationis, pulmonary embolism.

\section{Case report}

A 32-year-old primigravida in the $36^{\text {th }}$ week of gestation was admitted to the Dermatology Department with highly pruritic, polymorphous eruption of 3 weeks' duration. The medical history revealed labial infection with herpes simplex virus 4 weeks earlier.

Physical examination revealed erythematous urticarial papules and plaques forming concentric rings of targetlike appearance (erythema iris). The primary lesions were localized on the abdomen in the periumbilical area and extremities, especially on the hands and feet. Solitary vesiculobullous lesions and erosions were localized on distal parts of the extremities. There was no involvement of facial and mucous membranes.

The patient was treated with oral acyclovir at a dose of $400 \mathrm{mg}$ three times a day, dexamethasone $6 \mathrm{mg}$ i.v. once daily, oral antihistaminic (clemastine $1 \mathrm{mg}$ twice a day), and a topical high potency steroid. After the $6^{\text {th }}$ day of treatment, the patient was transferred to the Gynecology and Obstetrics Department, where she gave birth to a healthy female weighing $2590 \mathrm{~g}, 51 \mathrm{~cm}$ long, and 10 points in the Apgar Scale. The delivery occurred in the $37^{\text {th }}$ week of gestation and consisted of an uncomplicated, spontaneous vaginal delivery. The patient was treated with cefuroxime during the stay in the delivery room to avoid infection due to amniotic fluid leakage.
Postpartum, our patient developed generalized erythematous and papular skin lesions on the trunk, extremities, and face with exfoliation on the palmar surface of the hands and feet. There were bullous lesions and erosions localized on the extremities, abdomen, and trunk (Figures 1-4) prompting re-admission to the Dermatology Department. A biopsy was taken from the lesion on the patient's arm. Hematoxylin and eosin stain $(\mathrm{H}+\mathrm{E})$ revealed infiltrations of eosinophils in the dermal-epidermal junction and edema of the dermis. Direct immunofluorescence (DIF) of skin biopsy revealed positive linear deposits of complement C3C along the basement membrane zone (BMZ) (Figure 5). Indirect immunofluorescence (IIF) studies were conducted with monkey and guinea pig esophagus as substrate antibodies and IgG against a BMZ (1:80) of the epidermis were detected in the serum. These results confirmed the diagnosis of pemphigoid gestationis.

The patient was treated with intravenous dexamethasone at a dose of $8 \mathrm{mg}$ a day, oral antihistamine, cetirizine $30 \mathrm{mg}$ per day, and a topical medium potency steroid. On the third day of the treatment, the patient presented with chest pain, shortness of breath, and swelling of the right lower leg. To exclude a pulmonary embolism, the patient had computed tomography (CT) pulmonary angiogram, which revealed no abnormalities; there were no signs of deep venous thrombosis in duplex and color

Address for correspondence: Marta Wołek MD, Department of Dermatology, Jagiellonian University Medical College, 8 Skawinska Str., 31-066 Krakow, Poland, phone: +481243052 66, ext. 7400, e-mail: martawolek@o2.pl

Received: 30.07.2012, accepted: 2.12.2012. 


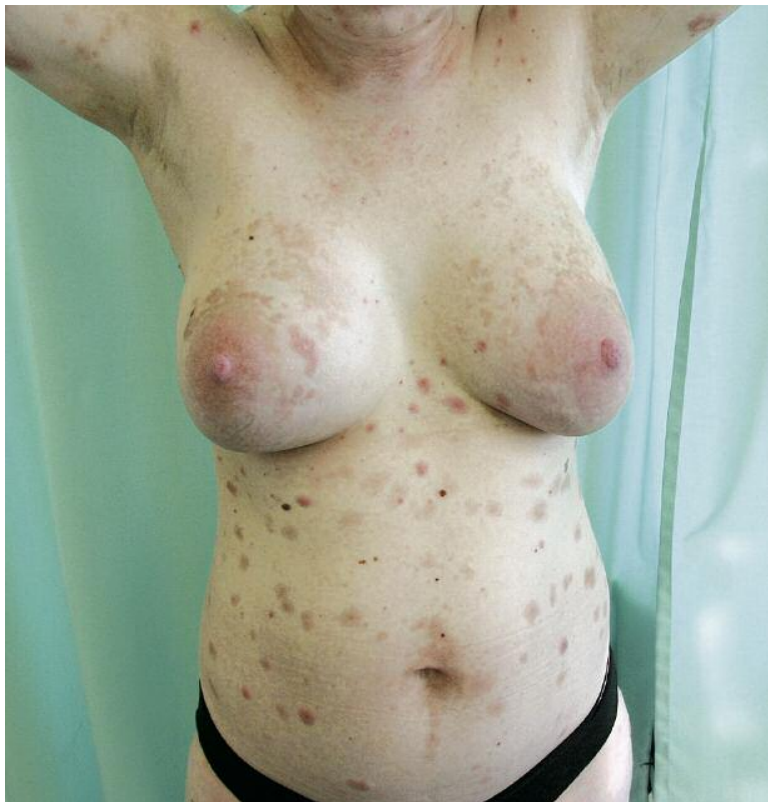

Figure 1. A view showing polymorphic eruption of herpes gestationis

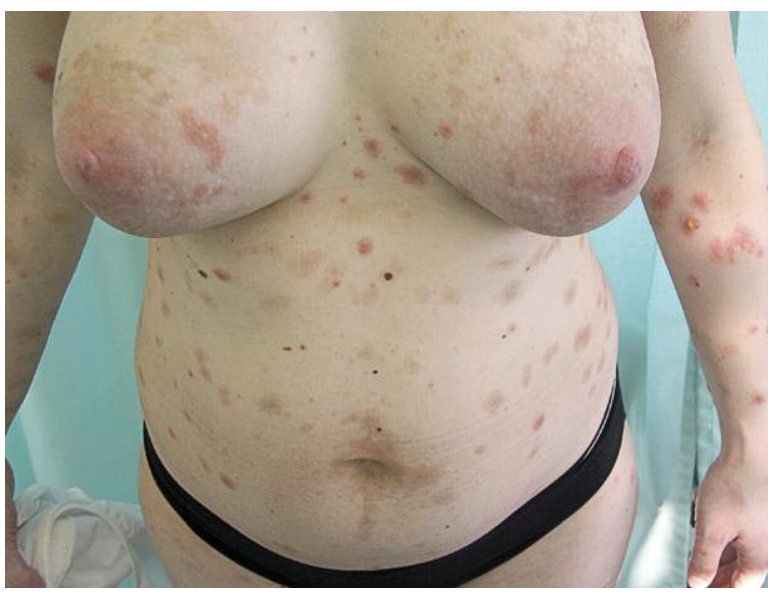

Figure 3. A close up view showing erythematous urticarial papules and plaques

Doppler ultrasound. Dyspnea and edema resolved after 2 days of treatment with furosemidum ( $40 \mathrm{mg}$ a day) and low-molecular heparin (enoxaparinum $60 \mathrm{mg}$ daily). The patient was discharged from the hospital in good condition and advised to continue treatment with cetirizine (20 mg per day) and a topical steroid. During a follow-up visit 3 days later, the patient revealed exacerbation of skin lesions with new bullae on the hands, forearms, and with generalized itching. The patient was treated for 2 months with methylprednisolone (in a decreasing dose from $32 \mathrm{mg}$ to $4 \mathrm{mg}$ per day), a topical medium potency steroid, and cetirizine at a dose of $20 \mathrm{mg}$ per day. The patient showed good response to the treatment and did not present new skin lesions 3 months later.

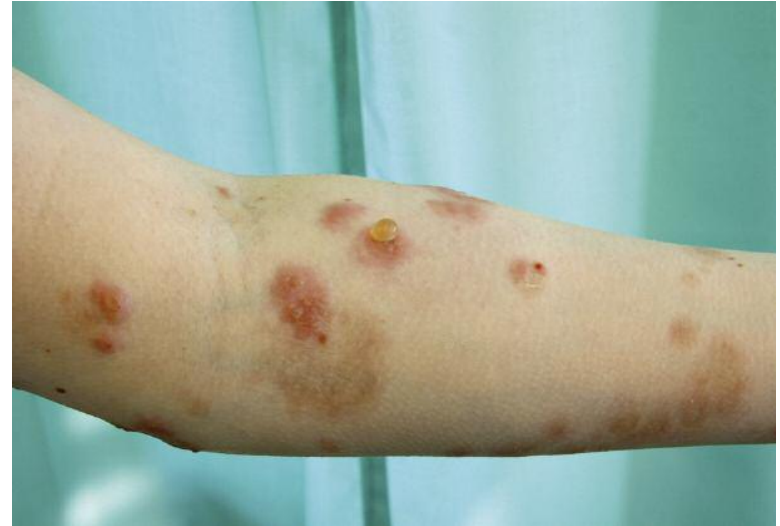

Figure 2. The bullous lesions and erosions localized on the left forearm

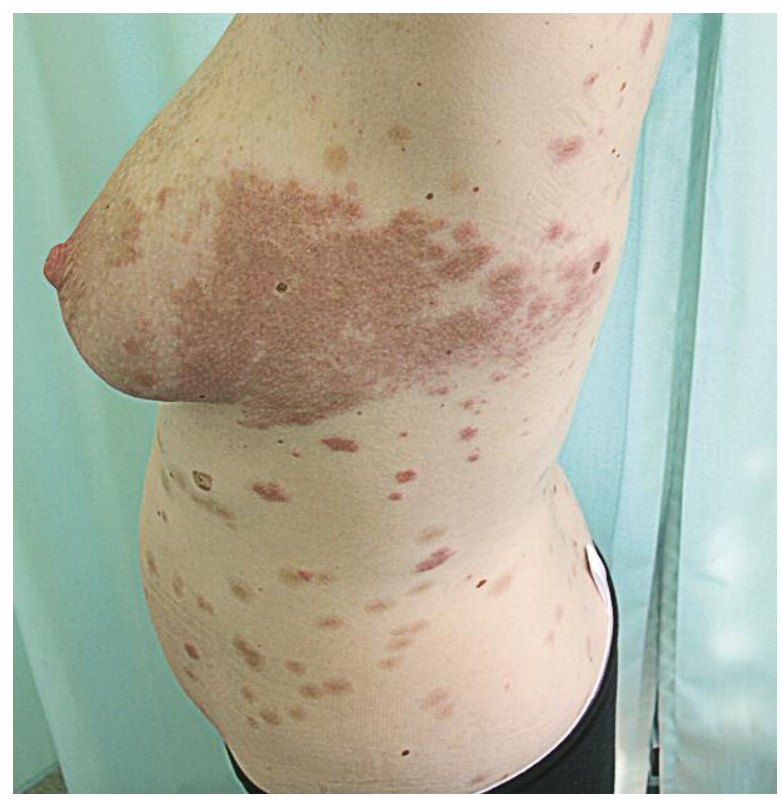

Figure 4. Erythematous urticarial plaque 4 days after partum

\section{Discussion}

Pemphigoid gestationis (synonyms: herpes gestationis, dermatitis multiformis gestationis) is a subepidermal blistering disease of pregnancy [1]. Pemphigoid gestationis has been estimated to occur in $1: 50000$ pregnancies and usually presents in the second or third trimester with exacerbation after partum [2-4]. This was the case for our patient. Initial symptoms appeared in the third trimester with deterioration after partum. The mother is affected more often than both the mother and the infant. In most cases, the disease is caused by circulating lgG1 immunoglobulin against hemidesmosomal protein bullous pemphigoid BP180 (BPAG2) type XVII collagen or BP230 $[2,5]$. The binding of IgG to the basement membrane leads 


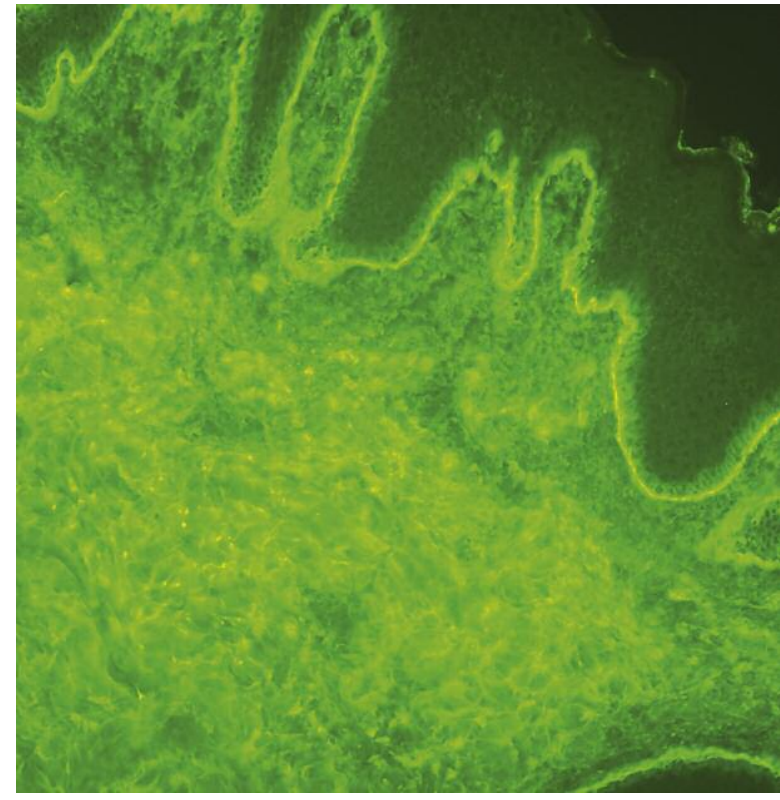

Figure 5. Direct immunofluorescence: linear deposits of complement C3 along the basement membrane zone

to the formation of subepidermal bullae and vesicles. Pemphigoid gestationis shows a genetic linkage to HLA-DR3 (80\% of the patients) and HLA-DR4 (53\%); 43-50\% express both MHC II genes [2, 3]. Subepidermal blister, dermal edema, and perivascular infiltrate of eosinophils, lymphocytes and histiocytes are observed in histopathological examination. Skin lesions in PG are polymorphous. The disease usually starts with pruritic papules or urticarial wheals on the abdomen (periumbilical distribution) and limbs. Some of lesions may produce target-like appearance and bulla, but not vesicles [4]. In our case, the initial location of the lesions, which were found on the abdomen, corresponded with the typical course of the disease. Symptoms may also be reminiscent of erythema multiforme, especially because of previous herpetic infection.

The diagnosis of pemphigoid is based on the DIF finding of C3 with IgG (in 25\% of cases) along the basement membrane zone in the skin. Pemphigoid gestationis is a selflimiting disease and can heal spontaneously after delivery [3, 6-9]. The aim of the treatment is to prevent new blister formation and relieve pruritus. Topical steroids and antihistaminic drugs constitute the first-line treatment; however, they are not sufficient in the majority of cases. Patients usually respond well to oral steroids (prednisolone $0.5 \mathrm{mg} / \mathrm{kg} / \mathrm{day}$ ). In the case of our patient, the treatment with oral antihistamine was not sufficient; therefore, we decided to start treatment with oral corticosteroids. Side effects must be kept in mind, especially an increased risk of thromboembolism during puerperium [10-12]. The risk of thromboembolism is also increased by the treatment with a systemic steroid. In more severe cases, when steroids are ineffective, the patient can be treated with immunoglob- ulin, immunosuppressants (methotrexate, cyclosporine), or plasmapheresis [2, 5].

Differential diagnosis of urticarial lesions includes erythema multiforme (especially if it is preceded by viral infection, e.g. herpes simplex), pruritic urticarial papules and plaques of pregnancy (PUPPP), or an exacerbation of other autoimmune bullous skin disorders.

Pemphigoid gestationis is a rare disease that requires proper diagnosis before the commencement of the treatment. Cooperation between the dermatologist, obstetrician, and neonatologist ensures that the patient will receive the best treatment with the lowest incidence of side effects.

\section{References}

1. Cozzani E, Basso M, Parodi A, et al. Pemphigoid gestationis post partum after changing husband. Int I Dermatol 2005; 44: 1057-8.

2. Lipozenčić J, Ljubojevic S, Bukvić-Mokos Z. Pemphigoid gestationis. Clin Dermatol 2012; 30: 51-5.

3. Bedocs PM, Kumar V, Mahon MJ. Pemphigoid gestationis: a rare case and review. Arch Gynecol Obstet 2009; 279: 235-8.

4. Kneisel A, Hertl M. Autoimmune bullous skin diseases. Part 1: Clinical manifestations. J Dtsch Dermatol Ges 2011; 9 : 844-56.

5. Dahiya K, Sharma D, Rajotia N, et al. Herpes gestationis. Taiwan J Obstet Gynecol 2009; 48: 425-7.

6. Takatsuka Y, Komine M, Ohtsuki M. Pemphigoid gestationis with a complete hydatidiform mole. J Dermatol 2012; 39: 474-6.

7. Patsatsi A, Vavilis D, Tsikeloudi M, et al. Refractory pemphigoid gestationis postpartum. Acta Obstet Gynecol Scand 2012; 91: 636-7.

8. Beard MP, Millington GW. Recent developments in the specific dermatoses of pregnancy. Clin Exp Dermatol 2012; 37: 1-4.

9. Woźniak K, Kazama T, Kowalewski C. MDA practical technique for differentiation of subepidermal bullous diseases: localization of in vivo-bound IgG by laser scanning confocal microscopy. Arch Dermatol 2003; 139: 1007-11.

10. Kęsik J, Zubilewicz T, Terlecki P. Prevention and treatment of venous thromboembolism in pregnancy. Przegl Menopauz 2010; 4: 275-9.

11. Regitz-Zagrosek V, Blomstrom Lundqvist C, Borghi C, et al. ESC Guidelines on the management of cardiovascular diseases during pregnancy: the Task Force on the Management of Cardiovascular Diseases during Pregnancy of the European Society of Cardiology (ESC). Eur Heart J 2011; 32: 3147-97.

12. Leung A, Bull T, Jaeschke R, et al. An official American Thoracic Society/Society of Thoracic Radiology clinical practice guideline: evaluation of suspected pulmonary embolism in pregnancy. Am J Respir Crit Care Med 2011; 184: 1200-8. 